\title{
17. The Gateway Review Process in Victoria
}

\section{Wayne Sharpe, Executive Manager, Gateway Unit, Department of Treasury and Finance, Victoria}

\section{Government policy rationale}

The Victorian Government spends billions annually on procuring infrastructure, information systems, real property, goods and services. Contemporary government procurement is now well accepted as a strategic management function requiring a commitment by departments, large and small, to effective procurement planning, innovative contracting strategies, active contract management and continuous improvement of procurement processes.

Implementing the Gateway Review Process is a key strategy for improving infrastructure and Information and Communication Technology (ICT) project development and delivery across government. The initial focus of the Gateway Review Process is on high-risk and medium-risk infrastructure, procurement and information technology/change management projects. The aim is to help departments ensure their investment is well spent, meets business objectives and achieves value for money outcomes.

Successful procurement projects depend on aligning service outcomes with project objectives in all phases of the procurement process. A project based on a sound and tested business case is more likely to achieve the planned benefits. The Gateway approach is a structured process that examines and confirms critical decision points from concept development through to project benefit evaluation. It probes the adequacy of the risk management framework to ensure that government procurement of construction, information technology and other services or projects is successfully delivered and that learnings are fed back, so that future projects can be managed better. It does this by enhancing the discipline of project delivery, for example, ensuring ongoing alignment of project objectives throughout the various stages of project development.

\section{Why a Gateway Review Process?}

The Gateway Review Process (GRP) is part of a broader Gateway initiative endorsed by the Victorian Government in March 2003. The initiative is a project management/development framework with three underlying objectives:

1. reduce budget/time overruns and scope changes in the delivery of initiatives by departments; 
2. improve alignment of initiatives with government strategic objectives and departmental corporate plans; and

3. better assessment of portfolio initiatives across government ('joined-up government').

Applying to major capital investments across the General Government sector, the Gateway Initiative consists of:

1. a multi-year strategy with a long-term view (5-10 years) of general Government sector asset investment projects or initiatives - aligned with the Victorian Government's strategic objectives;

2. development of consistent general Government sector project lifecycle guidance, with an initial focus on preparing better business cases to support asset investment proposals;

3. independent Gateway Reviews built around six key decision points in a project's lifecycle, with the aim of assuring successful project delivery; and

4. reporting focused on exceptions - identifying issues early enough to keep projects on track or get them back on track.

Evidence suggests that a primary cause of cost and time overruns in asset investment projects is a failure to identify and manage project risks at all stages of the project's lifecycle, including the particularly critical initial project planning stages. Gateway Reviews focus on addressing this issue.

The process has been developed and adopted by a number of major corporations and progressive governments.

\section{Characteristics of the Gateway Review Process}

The Gateway Review Process has been developed from tried and tested practices in industry and in the public sector in the United Kingdom ${ }^{1}$ and has the following key characteristics:

- it uses short, focused independent reviews;

- it uses a team of experienced and independent practitioners;

- it includes all key stakeholders;

- it is not an audit;

- it is undertaken in consultation with the project team; and

- it is confidential, and independent from the project approval process.

\section{Reactions to Gateway Review Processes in other jurisdictions}

The characteristics and benefits of the Gateway Review Process have ensured that it is well received throughout central civil government in the United Kingdom. The process has been successfully applied to projects undertaken by all its central civil departments including the Ministry of Defence. Departmental 
and industry reaction to the introduction has been extremely positive, with improvements to project delivery already demonstrated. Consultation with Gateway practitioners and other contacts in other jurisdictions has highlighted reactions and experiences.

\section{United Kingdom}

A number of benefits have emerged from the UK experience of implementing the Gateway Review Process, including increased assurance that the expected service delivery outcomes will result, delivery of projects within budget and time constraints, better management of risks inherent in projects, increased stakeholder satisfaction, and a snapshot of the procurement health of key projects for Senior Responsible Owners. ${ }^{2}$ Specific comments include:

- project owners who have received Gateway Review reports on their projects are supportive of the process and have benefited from recommendations contained in the Review report;

- it is of paramount importance to keep the Gateway Review Process separate from capital approval sections of government. Gateway Reviews are about 'helping projects succeed'. They are not part of the approval process;

- the Gateway Review Process must add value and support to projects, with the initial focus on high-risk projects; and

- the need to improve project delivery for projects which cut across departmental or jurisdictional boundaries is not well recognised or accepted (a common misperception is that 'all physical-build projects are low risk').

The cost of the Gateway Review Process to the central civil government in the UK is reported as approximately 0.01 per cent of the overall cost of projects. Office of Government and Commerce Value for money reviews have confirmed that average cost avoidance of 3-5 per cent are being achieved when best practice recommendations from review reports are implemented. The cost avoidance result from early implementation of actions needed for successful project delivery. For example a project with a total estimated investment of $\$ 100$ million would generate an average cost of $\$ 10,000$ to undertake a Gateway Review that could result in average cost avoidance of between $\$ 3$ and $\$ 5$ million.

\section{Practical benefits of Gateway Reviews}

Some Victorian Government departments and agencies have implemented excellent internal processes for obtaining better value for money spent on procurement. As a major procurer of projects, government recognises that processes can be further enhanced to improve project outcomes and services to the community. 
In other jurisdictions and in the private sector, experience has demonstrated that reviews at critical decision points in the procurement cycle add value and improve the outcomes of procurement and minimise the risk of project failure.

The Gateway Review Process involves targeted application of structured reviews, with the appropriate level of independence at critical decision points in project delivery. Implementation of Gateway Reviews can be expected to support a disciplined approach to the application of proven procurement processes leading to better outcomes.

Gateway Reviews will consolidate learning and procurement across the sector and give government and departmental Secretaries/CEOs confidence that high to medium-risk projects have had a level of expert independent review. The GRP provides three key benefits for government:

- a consistent, whole-of-government, disciplined process to help ensure that projects are delivered on time and within budget;

- increased confidence by departments, agencies and the Expenditure Review Committee of Cabinet in the health of a project; and

- an increased skill base across government through the development of review skills.

Implementing and operating the Gateway Review Process in Victoria will also help provide:

- successful projects delivered in a timely, efficient and appropriate way;

- best practice techniques in project delivery;

- lessons learned that are disseminated and incorporated into new projects;

- increased competence and valuable development opportunities for individuals involved in review teams; and

- enhanced project management capability within the Victorian public sector.

\section{Scope}

The Gateway Review guidelines apply to all new or existing high to medium-risk government projects that procure services, construction/property and information technology/change management projects.

At the earliest stages, if procurement is likely to be the chosen means of satisfying an identified need, departments and agencies should:

- assess the complexity and risk profile of the project;

- if high or medium risk, decide on the type of Gateway Review required and the timing; and

- schedule reviews to ensure discipline in the procurement process and an optimum value for money solution. 
The Gateway Review Process is not designed to duplicate existing approval processes, but should assist and enhance outcomes from these processes. The GRP generally serves a different purpose than internal approval or reviews processes.

\section{Establishing the Gateway Review Process}

As noted, the Gateway Review Process is based on established industry practice comprising short, structured, independent reviews at six key stages in the life of the project.

\section{Figure 1}

\section{Stage in project lifecycle}



The reviews are conducted for and reported to the Senior Responsible Owner. The reviews seek to identify issues to ensure the successful delivery of the project. 
The key to delivering objective high-quality reports is the independence of the review team from the project, and, in the case of high-risk projects, the independence of the review team from the project's department.

The review teams consist of senior government staff supplemented where necessary by external consultants. The interchange of senior staff between departments and agencies undertaking Gateway Reviews should improve project delivery across government.

\section{Setting up - the steps}

Several steps were involved in setting up support systems for the Gateway Review Process in Victoria. The plan involved establishing:

- an independent Gateway Unit with the role of developing, implementing and overseeing continuous improvement of the Gateway Review Process. The Gateway Unit has been initially incorporated into the Commercial Division of the Department of Treasury and Finance's organisational tree, with the operational and resourcing costs funded by Treasury, not on a cost recovery basis;

- a multi-department Gateway Supervisory Committee to oversee the implementation of the Gateway Review Process (including lessons learned and continuous improvement);

- Gateway Reviews for all projects classified as high risk for the 2003-04 Budget, with voluntary review of medium risk projects, and prioritised high risk projects to be considered at part of the 2004-05 budget process;

- feedback to the Gateway Supervisory Committee six times a year and quarterly to the Premier and the Treasurer on generic lessons learned from the findings of Gateway Reviews;

- encouragement of participation by senior experienced staff within the Victorian Public Sector as Gateway reviewers, particularly for high-risk projects;

- an accreditation and training regime to develop skills of Gateway reviewers in portfolio departments; and

- a database of accredited staff to participate in Gateway Reviews.

\section{What is required by departments?}

Government departments and agencies, managers, reviewers and particularly Senior Responsible Owners have a number of responsibilities in relation to the GRP. 


\section{Understanding the Gateway Process}

Government departmental and agency staff involved in procurement or project delivery should familiarise themselves with Gateway Review responsibilities and processes including:

- ensuring that the Project Profile Model (PPM) is completed and sent to the Gateway Unit when the project is first considered during the strategic phase or the business case generation phase;

- ensuring the conduct of reviews necessary under the Gateway Review requirements;

- considering review outcomes and recommendations in decision-making on the project; and

- understanding the independence of reports issued by the Gateway Review Teams.

Departments and agencies are expected to establish a mechanism for coordination with the Gateway Unit and to make all arrangements for the venue and equipment required for Gateway Reviews. In addition, departmental and agency staff involved in the Gateway Review Process should be familiar with government policies and guidelines relating to asset management, procurement, construction and information technology.

\section{Role of Senior Responsible Owner}

A Senior Responsible Owner, who is either a Senior Executive or Senior Manager, is to be nominated by the departmental Secretary or Chief Executive Officer for each procurement project subject to a Gateway Review. Senior Responsible Owners ensure that Gateway Review requirements are implemented for the project for which they are responsible. This includes:

- managing the implementation of the Gateway Review requirements;

- arranging for completion of projects' complexity rating using the PPM and classifying them;

- liaising with the departmental Secretary or the Senior Executive nominated as responsible for procurement on the risk rating for the project;

- providing a copy of the completed PPM risk assessment to the Gateway Unit; and

- coordinating Gateway Reviews of the project with the Gateway Unit.

\section{Selecting Gates for Review and nominating reviewers}

Gateway Reviews are conducted before key decisions are taken in the procurement cycle (see Figure 1 a more detailed version).

As noted, Senior Responsible Owners from departments and agencies are expected to communicate with the Gateway Unit at least six to eight weeks before a 
Gateway Review for all medium to high risk projects to ensure that the correct resources can be assembled and that the pre-Review work is completed.

The Gateway Unit assembles the review teams for all high-risk projects. The Unit will seek departmental nominations of potential review team members to be trained for medium and high risk projects. When trained, these people are considered accredited for Gateway Reviews of medium and high risk projects across other departments and agencies.

\section{Application of Gateway Reviews}

The primary purpose of the Gateway Review Process is to realise better value for government asset investment decisions. The process provides government and departmental Secretaries with assurance that:

- the best option to achieve a service objective is being pursued;

- independent review at key stages will help avoid potentially costly mistakes;

- the best available skills and experience are being deployed on the project;

- all stakeholders involved in the project fully understand the project status and the issues involved;

- the project can progress safely to the next stage of development or implementation;

- predictability of time and cost targets for the project is increased; and

- knowledge and skills are improved through participation in review teams.

\section{Project profile model}

The decision about conducting a Gateway Review is based on the complexity/risk assessment using a Project Profile Model (PPM). This assessment is done by departments.

The PPM is intended to provide a standard set of high-level criteria against which Senior Responsible Owners can assess the intrinsic characteristics and degree of complexity of a proposed procurement project, in order to establish the appropriate:

- control structures (including Gateway Reviews);

- risk profile and corresponding risk strategy; and

- design approach (for example, delivering the project in several increments or modules to help reduce complexity).

\section{Using the project profile model}

Three spreadsheets are provided by the Gateway Unit that are appropriate for:

1. Information technology/change management projects: all business change projects involving an IT element which, if not delivered, would significantly impact upon the project's ability to deliver its intended benefits. 
2. Property and construction projects: projects procuring property (existing or to be constructed) as a supply of works or service.

3. Other services: (e.g. environmental management, facilities management, property and estates advice etc).

Senior Responsible Owners should use the spreadsheet most appropriate for their project. ${ }^{3}$ The PPM should be used as a starting point in assessing the likely levels of risk associated with the project. It is a high level indicator, not an exhaustive project risk analysis model, although it can form the basis of a fuller project risk analysis. The model requires the Senior Responsible Owner to assess the project against a number of criteria to provide an overall score for the project. These initial scores will be validated by the Gateway Unit. The current approach is that:

- a total score of 20 or less indicates that the project is relatively low risk and Gateway Reviews will be managed from within the department or agency;

- a total score in the range of 21-40 indicates that the project is medium risk. Gateway Reviews for this category will require a review team leader, nominated by the Gateway Unit, who is independent of the department or agency. Review team members are sourced by the department or agency, outside of the project team for medium risk reviews; and

- a total score 41 or more indicates that the project is high risk and will require the review team leader and the review team members to be nominated by the Gateway Unit and to be independent of the department or agency.

It is important to stress that the PPM is designed as a guide to help the Senior Responsible Owner make their assessment. There may be issues that are not explicitly covered by the model but which affect the assessment. In particular, there may be other factors that increase the risk to the project and therefore warrant a higher rating. If in any doubt, Senior Responsible Owners should discuss these issues with the Gateway Unit.

Senior Responsible Owners are asked to notify the Gateway Unit (after confirming the PPM details with the departmental Gateway Coordinator) if the proposed project is medium or high risk. (The Gateway Unit does not record information for low-risk projects.)

The Gateway Unit needs a minimum of six to eight weeks from the receipt of the PPM to undertake the necessary planning and team selection. However, PPMs may be sent to the Unit in advance of this minimum period.

\section{Procurement types}

Gateway Reviews may be conducted at the six key decision points in the procurement cycle for all types of procurement, but the Gateway Process does not apply to all government projects, and not all Gateway Reviews are necessarily 
applied at all stages of projects. The need for and level of review required will be assessed on a complexity/risk basis. Core Gateway Reviews apply to specific procurement types, depending on the level of risk assessed via the PPM. Specific Gateway Reviews are required for different types of procurement. For instance, a post-completion Gateway Review would be appropriate for an information system project assessed as high risk with wide impacts across government, but may not be considered appropriate for a project procuring capital equipment assessed as low risk.

\section{Gateway Reviews - The Six Gates}

As noted earlier, there are six Gates, or key decision points, for reviewing projects. Gateway Reviews may be conducted for all types of procurement at any of the six key decision points (Gates) in the project (procurement) lifecycle: strategic assessment, business case, procurement strategy, investment decision, readiness for service and benefits evaluation. The detail in each Gateway Review is based on the results of workshops and tests on pilot projects.

\section{Strategic assessment (Gate 1)}

Gateway Review 1, strategic assessment, assesses whether the proposed procurement project is the best value means of servicing the identified need and whether it aligns with government and relevant departmental or agency strategic plans. To achieve this, the strategic assessment:

- confirms the need for the delivery of the service outcomes proposed;

- confirms that there is government and departmental/agency commitment to meeting the service need;

- ensures that the service and project objectives are fully enunciated;

- ensures investigation of all alternatives to procurement projects for meeting the perceived need;

- confirms whether a procurement project or program is the appropriate means of providing service outcomes;

- confirms that there has been a comprehensive investigation of alternative procurement methods including integration with service outcomes required of other departments or agencies ('joined-up' government);

- reviews plans for developing a business case, including financial and economic analysis, value management, risk management, stakeholder consultation, project management and change management; and

- confirms resources (including funding) are available to develop a sound case for government consideration for approval. 


\section{Business case (Gate 2)}

The business case review assesses whether the project options have been fully canvassed and evaluated, whether the recommended option is the best value solution, and whether government should proceed with it.

To achieve this, the business case review aims to:

- confirm that the business case is robust - that is, in principle it meets service needs, is affordable, achievable, that appropriate options have been explored, and that the project is likely to achieve value for money;

- establish that a feasibility/options study has been completed satisfactorily - including financial analysis meeting Treasury requirements, and that a preferred way forward has been identified;

- confirm that the implementation of the project is based on open and active communication with all major stakeholders represented;

- ensure that the major risks have been identified, that outline risk management plans have been developed and estimated costs of risks are included in the project budget;

- confirm that, for major asset initiatives, alternative delivery methods have been evaluated, for example. Partnerships Victoria private/public operation versus a government-funded project;

- confirm that the scope and project objectives are realistic, clear and unambiguous;

- establish that, in formulating the proposed project, impacts on suppliers and their ability to deliver has been fully considered;

- establish that robust plans for managing the next stage of the project are in place;

- establish that stakeholders were considered in formulating the project;

- review stakeholder opinions and establish that plans are in place for ongoing stakeholder consultation;

- review and validate assessment of assumptions made about the project; and

- review the analysis of economic, social and environmental impacts and confirm that the project meets government's current objectives.

\section{Procurement strategy (Gate 3)}

The procurement strategy review aims to propose the optimum methods for delivering the project within budget and time constraints and to allocate risks to the parties best able to manage them. This review is undertaken before committing to a procurement methodology and contracting system. To achieve this, the procurement strategy review aims to:

- confirm that the proposed project aligns with the business case and will deliver the service outcomes within the budget allocated; 
- ensure that the procurement strategy is robust and appropriate and has been established through authorised departmental/agency processes;

- establish that comprehensive plans for managing the project during the delivery process have been developed and are implemented;

- review risk management plans and establish that all major risks have been considered and plans for risk management are established, including budgetary provisions;

- ensure that supplier capacity and past performance have been realistically evaluated in developing the proposed procurement strategy;

- review benchmarks established to evaluate the project's success in delivering service outcomes;

- assess the appropriateness of the proposed contracting methodology and likelihood of its success in delivering the project and satisfying broader government policies;

- ensure the procurement method is in place; and

- ensure the site has been secured and all other pre-procurement actions are in hand.

\section{Tender decision (Gate 4)}

The tender decision review assesses whether the business case is valid once costs are established. The review also assesses whether the investment decision process was conducted with due probity and fairness to tendering parties.

To achieve this, the tender decision review aims to:

- confirm the business case and benefits plan when the bid information is confirmed;

- check that all necessary statutory and procedural requirements were followed throughout the procurement process;

- confirm that the recommended contract decision - if properly executed within a standard lawful agreement - is likely to deliver the specified outputs/outcomes on time, and within budget and will provide value for money;

- ensure that management controls are in place to manage the project through to completion;

- ensure there is continuing support for the project;

- confirm that the approved procurement strategy has been followed;

- confirm that the development and implementation plans of the client and the supplier or partner are sound and achievable;

- check that the business is prepared for developing any new processes where required, and has prepared for implementation, transition and operation of new services/facilities; 
- confirm that there are plans for risk management, issue management and change management (technical and business) and that these plans are shared with suppliers; and

- confirm that the technical implications, such as 'buildability' for construction projects and, for IT-related projects, the impact of e-government frameworks, have been addressed.

\section{Readiness for service (Gate 5)}

The readiness for service review assesses the state of readiness to commission the project and implement the change management required.

To achieve this, this pre-commissioning review is designed to:

- check that the current phase of the contract and all documentation is properly completed;

- ensure that the contractual arrangements are up-to-date;

- check that the business case is still valid and unaffected by internal and external events or changes;

- check that the originally projected business benefit is likely to be achieved;

- confirm that there are processes and procedures to ensure long-term project success;

- confirm that all necessary testing is done to the client's satisfaction (e.g. commissioning of buildings, business integration and user acceptance testing) and that the client is ready to approve implementation;

- check that there are feasible and tested contingency and reversion arrangements;

- ensure that all ongoing risks and issues are managed effectively and do not threaten implementation;

- evaluate the risk of proceeding with the implementation if there are any unresolved issues;

- confirm the business has the necessary resources and that it is ready to implement the services and the business change;

- confirm that the client and supplier implementation plans are still achievable;

- confirm that there are management and departmental controls to manage the project from implementation to operation;

- confirm that all parties have agreed plans for training, communication, roll-out, production release and support as required;

- confirm that all parties have agreed plans for managing risk;

- confirm that there are reciprocal plans for managing the working relationship, with reporting arrangements at appropriate levels on both the department/agency and the supplier side; and

- check that lessons for future projects are identified and recorded. 


\section{Benefits evaluation (Gate 6)}

The benefits evaluation review assesses whether the benefits expected in the business case have been achieved. The review will ensure that post-completion and post-occupancy reviews are conducted and the findings communicated, to improve future projects.

To achieve this, the benefits evaluation review aims to:

- confirm that post-completion review and post-occupancy evaluation have been carried out and the findings disseminated to participants in the procurement chain;

- assess whether the project has met business case goals and whether claimed operational benefits have been realised;

- review the adequacy of funding arrangements for ongoing operation and management of the project;

- establish the benefits of applying Gateway Reviews and government procurement processes to the project;

- identify improvements that might be made to existing procurement processes, as a result of experience from this project;

- if ongoing contract management is required, review the adequacy of client and supplier resources for the task;

- identify any deviations from the business case for the project;

- confirm that plans are in place for future renewal of the procurement project;

- review the adequacy of risk management plans for the project;

- review the adequacy of project change management plans;

- review the extent to which the project met stakeholder requirements; and

- review the project one year after it has been completed, and on a three-year cycle for the life of the project thereafter, to ensure the ongoing benefits of the project.

\section{Gateway Review Reporting and Support}

\section{Review Guidelines}

Guidelines to assist the review team conduct a review are available for each Gateway Review. The Gateway Unit can provide documentation and presentations to assist at any particular Gateway or for the general review process.

\section{Reports}

As project personnel, clients and other stakeholders may be interviewed about issues arising in the Review, review teams produce a short report summarising the review activities undertaken and the conclusions of the team about the health of the project. Review reports are provided to the Senior Responsible Owner only. If a copy of the report is sought it is at the discretion of the Senior 
Responsible Owner if he or she releases the report. The report will not be released or circulated by the Gateway Review Team of the Gateway Unit.

Gateway Review reports provided to the Senior Responsible Owner, like all other government documents, are subject to the Freedom of Information Act 1988. Freedom of Information (FOI) requests for Gateway Review reports will be handled through the Senior Responsible Owner's department and not through the Gateway Review Unit. Certain information contained within the Gateway Review reports may be excluded from release as part of the various exemptions in the FOI Act.

The Gateway Unit retains a copy of the each Review report, to compile lessons learned for reporting generically to the Gateway Supervisory Committee and back to relevant departments or agencies. The Gateway Review Process uses Red, Amber and Green (RAG Status) classifications for assessing projects at each Gate:

Red - To achieve success the project should take action immediately.

Amber - The project should go forward with actions on recommendations to be carried out before the next Gateway Review of the project.

Green - The project is on target to succeed but may benefit from the uptake of recommendations.

\section{Review Team Status}

Review teams have no decision-making powers. All decisions arising from review recommendations are made by the Senior Responsible Owner.

\section{Supporting Gateway Reviews}

\section{Importance of the review team}

Any Gateway Review relies substantially on the independent expertise of the review team. Reviewers are required to have high-level skills, through training and extensive or relevant experience of aspects of the projects under review.

Review team members will be chosen from the private sector or other government jurisdictions when necessary to supplement skills and experience within the Victorian public sector and the Gateway Unit. Registration of Interest advertisements and other methods are used to compile a database of experienced private sector people to supplement review teams.

\section{Training reviewers}

Training is provided on 'Preparing to carry out Gateway Reviews' and 'Leading Gateway Reviews'. The training is designed to equip participants with the knowledge and skills to plan, prepare and undertake Gateway Reviews as team members and team leaders of high, medium and low risk reviews. Training is 
delivered by a external organisation, utilising modified OGC Gateway Training material from the UK. For details on how to undertake training please see the Gateway Review website.

\section{Accreditation and skills - reviewers and team leaders}

There are different levels of training for review team members and team leaders. The database of accredited departmental and agency reviewers as well as the private sector reviewers (established by the Gateway Unit) will include details of their skill base and experience. New nominees will be assessed and considered for inclusion on the database of reviewers. The reviewers details in the database are subject to the Privacy Act and are only utilised by the Gateway Review Unit. Nominees for Gateway Review team membership are expected to meet certain minimum requirements.

This outline recognises three levels of skills or expertise:

Level I: Awareness. The participant is able to understand the key issues and their implications for the client, and ask relevant and constructive questions on the subject.

Level 2: Knowledge. The participant has a detailed knowledge of the subject and is capable of providing guidance and advice to others.

Level 3: Expert. The participant has extensive and substantial practical experience and applied knowledge of the subject.

The Gateway Unit will advise nominees and their departments or agencies of their inclusion on the database.

(i) Team leaders - There are particular requirements for team leaders of reviews of medium and high-risk projects.

(a) For high-risk projects, team leaders are:

- typically executive level in the public sector and the equivalent in the private sector; 4

- $\quad$ substantially experienced (minimum of 15 years), with a background in managing business aspects of major procurement projects and/or programs with a typical value of $\$ 50$ million or more;

- familiar with handling major strategic initiatives and managing business change;

- excellent communicators; and

- experienced in connection with sensitive and complex or unusual projects.

(b) For medium-risk projects, team leaders are:

- minimum level - Victorian Public Sector level 5; 
- $\quad$ substantially experienced (minimum of 10 years), with a varied background, particularly in procurement or project/program management typically valued at \$20 million; and

- excellent communicators.

(ii) Team members - Team members need a certain level of project management experience and/or experience in business change, business analysis or other operational or procurement areas. They include:

(a) Project managers with the following experience:

- $\quad$ professional project managers, who are accredited in Prince 2 (UK Office of Government Commerce project management methodology) or PMBOK (the Project Management Body of Knowledge Guide);

- successful managers of significant projects/programs or procurements;

- good team players and communicators; and

- $5^{+}$years relevant experience.

(b) Business analysts with:

- in-depth financial assessment evaluation experience; and

- experience in preparing business cases, strategic assessments and business requirements.

(c) Specialists in management in the following areas (minimum five years experience):

- business change;

- electronic documents and records;

- facilities; and

- construction and property.

(d) procurement professionals (minimum 5 years experience):

- professional procurement practitioners;

- with experience in managing contractual relationships; and

- with responsibilities for best procurement practice.

(e) operations experience (minimum five years) in:

- operations support;

- property portfolio management; and

- service management.

No fees are paid to departmental/agency personnel for participation in review teams. 


\section{Conclusion}

Since the introduction of the GRP in 2003, over 90 projects with a total estimated investment of over $\$ 14$ billion have had more than 120 reviews completed.

Of the SRO's that have provided feedback (greater than 80 per cent), all agreed that the Gateway Review undertaken on their project was beneficial and will impact positively on the outcome of their project.

A recently completed independent review of the Gateway Initiative, has also determined that:

- the Initiative's first component, the Gateway Review Process, helps identify problems early to allow time for their remedy;

- the Initiative's second component, the Multi-Year Strategy, has improved the alignment of asset projects with Government strategic objectives and department plans but, importantly, the lack of sharing of the Multi-Year Strategies among Departments is weakening Gateway's objective of better whole-of-government planning;

- the Initiative's third components, Business Cases, are useful decision-making tools for Departmental Secretaries and Government to determine how asset procurement should best occur; and

- based on the lessons learned from over 100 Gateway Reviews a publication entitled 'Commonly Identified practices that limit project success' ${ }^{*}$, has been produced to share generic lessons learned.

\section{Annex: Commonly Identified practices that limit project success can be downloaded from:}

http://www.gatewayreview.dtf.vic.gov.au/CA256EF40083ACBF/WebObj/Gateway LessonsLearned/\$File/Gateway\%20Lessons\%20Learned.pdf

\section{Glossary}

Gateway Review: Review by a small team of people, independent of the procurement project, undertaken at key decision points (Gates) in the project (procurement) lifecycle

Government department: The terms government 'department', 'department' or 'funding department' are used interchangeably. Agencies are included in the Gateway Process.

Infrastructure: Fixed assets that support economic and social development in a fundamental way.

Milestone: Significant events or outcomes that mark the progress of a project.

Monitoring: Process of regularly collecting information to review performance against specified criteria. 
Outcome: Measurement against specified criteria of the extent to which objectives are achieved.

Project profile model: The project profile model (PPM) is a high-level assessment of the risk of a procurement activity. It provides an indication of the project's complexity/risk rating: high (category 3), medium (category 2) or low (category 1).

Procurement: Process involving all activities following the decision that goods, assets, facilities or services are required. It involves defining the scope, the acquisition and the disposal of goods, assets, facilities and services.

Project: An undertaking with a defined beginning and objectives by which completion is identified. A project may be completed using one or a number of contracts.

Risk management: A structured methodology for identifying and analysing potential risks and implementing an appropriate plan to manage them.

Senior Responsible Owner: The Senior Responsible Owner is a generic title for the senior individual who takes personal responsibility for the successful outcome of a program or project.

Service provider: Includes contractors, sub-contractors, consultants, professional service contractors, suppliers, agents and employers who provide goods, assets, facilities or services to a client.

Skill development: Any work-related learning or training activity that results in enhanced skills, knowledge and aptitude to perform a job.

Training: The development of skills, knowledge and aptitude to perform a job.

Value for money: Value for money is determined by considering all factors relevant to a particular purpose. It includes experience, quality, reliability, timeliness, service, capital costs, whole-of-life costs, innovation and value-adding components such as meeting government's economic, social and environmental policies.

\section{ENDNOTES}

${ }^{1}$ Office of Government Commerce. For more information, see www.ogc.gov.uk.

2 The Senior Responsible Owner is a generic title for the senior individual who takes personal responsibility for the successful outcome of a program or project.

${ }^{3}$ Please note that the first two spreadsheets are applicable to IT and construction/property management projects procuring services. While such projects may be seeking to pass responsibility to service providers for some of the criteria addressed within the PPM (e.g. the degree of innovation used) these factors will still be fundamental to the ultimate success or otherwise of the project and will need to be monitored throughout the project lifecycle by the Senior Responsible Owner.

4 This will vary depending on the requirements of the Gateway Review under consideration. 